\title{
PRODUÇÃO INTEGRADA E CONVENCIONAL DE PÊSSEGOS cv. MARLI ${ }^{1}$
}

\author{
JOSÉ LUIS DA SILVA NUNES ${ }^{2}$, DENIS SALVATI GUERRA ${ }^{3}$, CLAITON ZANINI ${ }^{4}$, VINICIUS GRASSELLI ${ }^{5}$, FABIANO \\ ARGENTA $^{6}$, HELENO FACCHIN ${ }^{7}$, GILMAR ARDUINO BETTIO MARODIN ${ }^{8}$
}

\begin{abstract}
RESUMO - O cultivo de pessegueiros é uma atividade de grande importância econômica no Sul do Brasil, onde se destaca o Estado do Rio Grande do Sul como maior produtor brasileiro. Um dos aspectos mais importantes na produção de alimentos da atualidade é a redução no uso de agroquímicos, com menor contaminação do ambiente e riscos reduzidos de resíduos. Este trabalho visou a comparar os sistemas de Produção Convencional (PC) e Integrada (PI) de pêssegos e foi realizado no ano de 2001, no município de São Jerônimo - RS, latitude 3005'52” S, longitude 51³9'08” W e altitude de 46 metros. Áreas de um pomar comercial da cv. Marli foram avaliadas em relação às principais práticas de manejo da planta e do solo, controle fitossanitário, aspectos econômicos, bem como à qualidade da fruta. Na área conduzida sob PI, foram utilizadas as práticas de manejo preconizadas pelas Normas de Produção Integrada de Pêssegos (NPIP) e, na área conduzida no sistema de PC, as plantas foram manejadas de acordo com as práticas comumente utilizadas pelo produtor. A produção de pêssegos, em ambos os sistemas, não foi afetada. Na área de PI, houve menor número de pêssegos por planta; entretanto, as frutas apresentaram maior peso médio. A maioria dos pêssegos da PI foram classificados como CAT I (diâmetro superior a $57 \mathrm{~mm}$ ). As frutas produzidas na PC são, na maioria, de CAT II (de 48 a $57 \mathrm{~mm}$ ). A qualidade pós-colheita não apresentou diferenças em relação à acidez, firmeza e cor. Com base nestes resultados, podemos concluir que é possível produzir pêssegos de qualidade com produtividade no sistema de PI.
\end{abstract}

Termos para indexação: Prunus persica, Sistemas de Produção, qualidade.

\section{INTEGRATED AND CONVENTIONAL PRODUCTION OF PEACH cv. MARLI}

\begin{abstract}
The peach-trees cultivation is a very economic important activity in South of Brazil, where highlights the State of Rio Grande do Sul as the largest Brazilian producer. One of the most important aspects in actuality is the food prodution with reduction of agrochemicals components, reducing the risks of environment contamination. This work aimed to compare the Conventional Production systems (PC) and Integrated systems (PI) of peaches cultivation and took place in the year of 2001, in the county of São Jerônimo - RS, latitude 3005'52” S, longitude 51 39 '08” W and altitude of 46 meters. Commercial orchand areas of cv. Marli was evaluated regarding the mains handling practice of plant and soil, phytosanitary control, economic aspects, as well as fruit production and quality. In the area under PI, were used the handling practices praised by the Norms of Production Integrated of Peach (NPIP) and in the area conducted the PC system, the plants were managed according to the practices usually used by the producer. The peaches production, in both systems, was not affected. The area of PI, occured smaller number of peaches per plant, however the fruit was larger middleweight. Most peaches of PI were classified like CAT I (diameter up to $57 \mathrm{~mm}$ ). The fruits produced in the PC were of the CAT II (48 to $57 \mathrm{~mm}$ ). Regarding to titratable acidity, flesh firmness and epidermal color there was no difference between peaches from either system. With these results, we can conclude that is possible to produce quality peaches with productivity in PI's system.
\end{abstract}

Index terms: Prunus persica, production sistems, quality.

\section{INTRODUÇÃo}

A área cultivada com pessegueiros no Brasil é superior a 20.000 hectares, com uma produção na ordem de 160.000 t.ano- ${ }^{1}$ (Fachinello et al., 2001). Deste total, 14.000 hectares são cultivados no Rio Grande do Sul, onde está localizada mais de $70 \%$ da produção nacional, sendo plantadas cultivares para conserva e para consumo in natura, com uma produção de 80.000 t.ano- $^{1}$, produzidas em quatro regiões distintas Pelotas, Grande Porto Alegre, Serra Gaúcha e região da Campanha (Marodin \& Sartori, 2000).

A tendência dos mercados mundiais é, além das qualidades interna e externa das frutas, também exigir o controle sobre todo o sistema de produção, incluindo a análise de resíduos nas frutas e estudo sobre o impacto ambiental, ou seja, o sistema de produção deve permitir a rastreabilidade de toda a cadeia produtiva (Fachinello et al., 2000). O Sistema de Produção Integrada parece ser a melhor alternativa para se obter uma produção de boa qualidade, pois procura equacionar os problemas, através de uma visão multidisciplinar, obtida a partir de métodos ecologicamente mais seguros, minimizando os efeitos colaterais indesejáveis do uso de agroquímicos, aumentando a proteção ao meio ambiente e estabelecendo um novo equilíbrio com o agroecossistema, sem prejudicar a saúde humana (Fachinello et al., 2001).

Na região Sul do Brasil, dois programas de Produção Integrada de Frutas vêm-se destacando: o de pêssegos e o de maçã. O projeto de pesquisa de Produção Integrada de Frutas de Pêssegos (PIP) foi implantado no RS em 1999 e é conduzido em pomares comerciais de pêssegos nas regiões produtoras (Farias, 2002).

O objetivo deste trabalho foi comparar os sistemas de Produção Convencional e Integrado em relação à produtividade e qualidade, visando à ampliação dos conhecimentos sobre a sustentabilidade do sistema de Produção Integrada de Frutas, com ênfase nos aspectos produtivos.

\section{MATERIALEMÉTODOS}

O experimento foi realizado na safra 2001-2002, em pomar comercial de 11 anos de idade, de propriedade dos Irmãos Bettio, localizado no município de São Jerônimo - RS, solo classificado como Argissolo Vermelho distrófico, de textura argilosa, situado à latitude $30^{\circ}$ $05^{\prime} \mathrm{S}$ e longitude $51^{\circ} 39^{\prime} \mathrm{W}$, a $46 \mathrm{~m}$ de altitude. Tomou-se como área experimental um pomar de pessegueiros da cv. Marli. O pomar foi dividido em áreas com aproximadamente 1 ha cada, onde foram desenvolvidos os sistemas de Produção Integrada (PI) e Produção Convencional (PC), distanciados cerca de 500 metros. Os dados climáticos do ano constam na Tabela 1.

$\mathrm{Na}$ área conduzida sob sistema de PI, foram aplicadas as práticas recomendadas pelas Normas de Produção Integrada de Pêssego (NPIP),

\footnotetext{
${ }^{1}$ (Trabalho 032/2004). Recebido: 19/04/2004. Aceito para publicação: 15/09/2004. Trabalho financiado pelo MAPA/CNPq.

${ }^{2}$ Doutorando em Fitotecnia - UFRGS, CP 15100, CEP 91501-970, Porto Alegre-RS, e-mail: tobnunes@ig.com.br.

${ }^{3}$ Doutorando em Fitotecnia - UFRGS, CP 15100, CEP 91501-970, Porto Alegre-RS, e-mail: d.s.guerra@ibest.com.br.

${ }^{4}$ Mestrando em Fitotecnia - UFRGS, CP 15100, CEP 91501-970, Porto Alegre-RS, e-mail: claiton@ terra.com.br.

${ }^{5}$ Estudante de Graduação - UFRGS, CP 15100, CEP 91501-970, Porto Alegre-RS, e-mail: vgrasselli@zipmail.com.br.

${ }^{6}$ Estudante de Graduação - UFRGS, CP 15100, CEP 91501-970, Porto Alegre-RS, e-mail: fargenta@ zipmail.com.br.

${ }^{7}$ Estudante de Graduação - UFRGS, CP 15100, CEP 91501-970, Porto Alegre-RS, e-mail: facchin@engineer.com.br.

${ }^{8}$ Prof. Dr. do DHS- FA/UFRGS, CP 15100 , CEP 91501-970, Porto Alegre-RS, e-mail: marodin@ vortex.ufrgs.br.
} 
TABELA 1 - Temperaturas máxima, média e mínima, precipitação, umidade relativa e número de horas de frio iguais ou abaixo de $7^{\circ} \mathrm{C}$, no município de Eldorado do Sul, 2001.

\begin{tabular}{|c|c|c|c|c|c|c|}
\hline \multirow[t]{2}{*}{ Meses } & \multicolumn{3}{|c|}{ Temperatura } & \multirow{2}{*}{$\begin{array}{l}\text { Precipitação } \\
\text { (mm) }\end{array}$} & \multirow{2}{*}{$\begin{array}{l}\text { UR } \\
(\%)\end{array}$} & \multirow{2}{*}{$\frac{\text { Número de horas de frio }}{7^{\circ} \mathrm{C}}$} \\
\hline & Máx & Méd & Mín & & & \\
\hline Janeiro & 30,3 & 24,2 & 18,3 & 171,3 & 83 & - \\
\hline Fevereiro & 30,4 & 25,0 & 20,9 & 121,5 & 82 & - \\
\hline Março & 30,0 & 24,2 & 19,9 & 143,7 & 81 & - \\
\hline Abril & 25,6 & 20,2 & 15,8 & 290,9 & 84 & - \\
\hline Maio & 20,7 & 15,4 & 10,4 & 36,1 & 83 & 2 \\
\hline Junho & 21,0 & 15,1 & 9,9 & 118,0 & 84 & 12 \\
\hline Julho & 19,4 & 13,6 & 8,2 & 227,6 & 84 & 34 \\
\hline Agosto & 24,2 & 17,0 & 11,0 & 36,4 & 79 & 15 \\
\hline Setembro & 21,8 & 16,6 & 11,8 & 261,7 & 81 & 18 \\
\hline Outubro & 25,7 & 20,3 & 15,7 & 63,6 & 79 & - \\
\hline Novembro & 27,3 & 21,0 & 14,9 & 171,4 & 75 & - \\
\hline Dezembro & 27,9 & 21,9 & 16,2 & 92,7 & 77 & - \\
\hline Médias & 25,4 & 19,5 & 14,4 & 144,6 & - & - \\
\hline Totais & - & - & - & 1735,0 & - & 81 \\
\hline
\end{tabular}

Versão II (Normas, 2001). Na área conduzida sob sistema de PC, o produtor utilizou as práticas comumente usadas na região.

Para a avaliação da qualidade na colheita, foram coletadas amostras de 20 frutas por planta e realizadas determinações de firmeza da polpa, de acidez total titulável (ATT) e sólidos solúveis totais (SST), conforme descrito por Seibert (1997). Essa etapa foi realizada no Laboratório de Pós-Colheita do Departamento de Horticultura e Silvicultura da Faculdade de Agronomia/UFRGS. Para avaliação de coloração, foram colhidos 10 frutos de cada planta e submetidos ao medidor de cores marca Minolta modelo CR300, determinando a intensidade e a pureza da cor vermelha da superfície das frutas.

O delineamento experimental utilizado nas avaliações a campo foi o inteiramente casualizado, com 10 plantas selecionadas, sendo cada planta uma unidade experimental. Os dados foram submetidos à análise de variância e a comparação de médias pelo teste $t(P \leq 0,05)$, com transformação das observações segundo raiz de $(x+1)$. A análise das variáveis estudadas foi executada pelo programa Sanest.

\section{RESULTADOS E DISCUSSÃO}

Na safra 2001, não se verificou diferença estatística na quantidade de frutas por planta (kg.planta- ${ }^{1}$ ) e na produtividade (t.ha- ${ }^{1}$ ). O sistema de PI proporcionou número de frutas/planta significativamente inferior ao número de frutas/planta produzidas em pomar conduzidos no sistema de PC (Figura 1). Atribui-se essa diferença principalmente ao raleio que foi realizado de forma metódica nas plantas da área da PI, conforme estabelecido pelas Normas da Produção Integrada de Pêssegos (NPIP), e que foi realizado na área da PC. Segundo Raseira et al. (1998), o raleio é uma das práticas culturais menos empregadas pelos produtores, em especial os de frutas de caroço, apesar de sua importância para as características comerciais da fruta, as quais, em última instância, influenciam no resultado econômico da exploração. Para Sartori (2001), o maior peso das frutas se deve à menor carga das plantas e à maior relação folha/fruta, em torno de 30 a 40 folhas para cada fruta.

Quanto ao peso médio, os pêssegos provenientes do pomar conduzido no sistema de PI apresentaram peso superior aos colhidos no pomar de PC, efeito devido ao raleio praticado na PI, o que revela a importância dessa prática (Figura 1). Mesmo assim, devido às altas temperaturas, favoráveis ao aparecimento de pragas e doenças (Tabela 1) e a problemas fisiológicos (período de estiagem prolongado no período de crescimento das frutas) que provocaram queda de frutos, as frutas restantes na PC tiveram um peso médio acima de 100 gramas, o que é considerado normal para cv. Marli.

Estes dados diferem dos obtidos por Botton et al. (2000), que relatam que, na região da Serra Gaúcha, na safra de 1999, as áreas conduzidas no sistema de PI produziram mais que as áreas no sistema de $\mathrm{PC}$, com frutas de peso médio semelhante. O mesmo ocorreu na região de Pelotas, na mesma safra, onde não foram observadas diferenças estatísticas em relação ao peso médio dos pêssegos produzidos em sistema de PI e PC (Nachtigall et al., 2000). Porém, conforme Fachinello et al. (2002), em pomares da cv. Diamante na região de Pelotas, safra de 2001, a produção foi maior no sistema de PI, com frutas de peso médio superior.

Com relação à classificação quanto ao calibre das frutas, o pomar conduzido sob o sistema de PI apresentou um número superior de frutas classificadas como CAT I ( $\geq 57 \mathrm{~mm}$ ). Na CAT II (de 48 a $57 \mathrm{~mm}$ ), houve diferença significativa entre os dois sistemas, sendo superior na PC. Na CAT III ( $\leq 48 \mathrm{~mm})$, houve maior proporção no sistema de PC (Figura 2). A explicação para o maior tamanho das frutas do sistema de PI foi a maior intensidade de raleio das frutas, conforme já discutido. Verificase que quase $70 \%$ dos pêssegos produzidos sob o sistema de PI pertencem a CAT I, tendo 25,3\% das frutas na CAT II e apenas 5,5\% das frutas na CAT III. Na PC, apenas $28 \%$ pertencentes a CAT I, $42,1 \%$ na CAT II e um percentual de $30 \%$ na CAT III, maior do que o da CAT I. Estes dados se assemelham aos obtidos por Fachinello et al. (2002), na região de Pelotas, onde a avaliação de qualidade das frutas indicou que houve aumento de frutas na categoria CAT I nas três safras (1999, 2000 e 2001), no sistema de PI. Porém, diferem dos obtidos por Botton et al. (2000), na safra de 1999, na região da Serra Gaúcha, onde não foram observadas diferenças significativas de categoria entre os dois sistemas.

Não houve diferenças significativas na intensidade e pureza da coloração vermelha da superfície das frutas entre os sistemas (Figura 3). Segundo Raseira et al. (1998), uma das práticas de manejo das plantas que mais influencia na coloração dos frutos é a poda verde. Esta prática é realizada quando a planta está em pleno desenvolvimento vegetativo, visando a melhorar a insolação e a aeração no interior da copa e aumentar a frutificação nas camadas inferiores dos ramos. Mesmo tendo sido realizada com uma semana de antecedência na PI, garantindo um período de maior exposição das frutas à luz solar, isto não foi suficiente para aumentar a coloração das frutas em relação à PC.

Quanto à firmeza de polpa das frutas, não houve diferenças significativas entre os sistemas (Figura 3). A firmeza de polpa é uma das transformações mais pronunciadas durante a maturação dos pêssegos e está vinculada à velocidade deste processo. Fachinello et al. (2002) afirmam que, na safra de 2001, em pomares na região de Pelotas, as frutas do sistema PI apresentaram, na colheita, maior firmeza de polpa do que o sistema PC, contrastando com resultados obtidos neste estudo.

A acidez total titulável (ATT) dos pêssegos não apresentou 

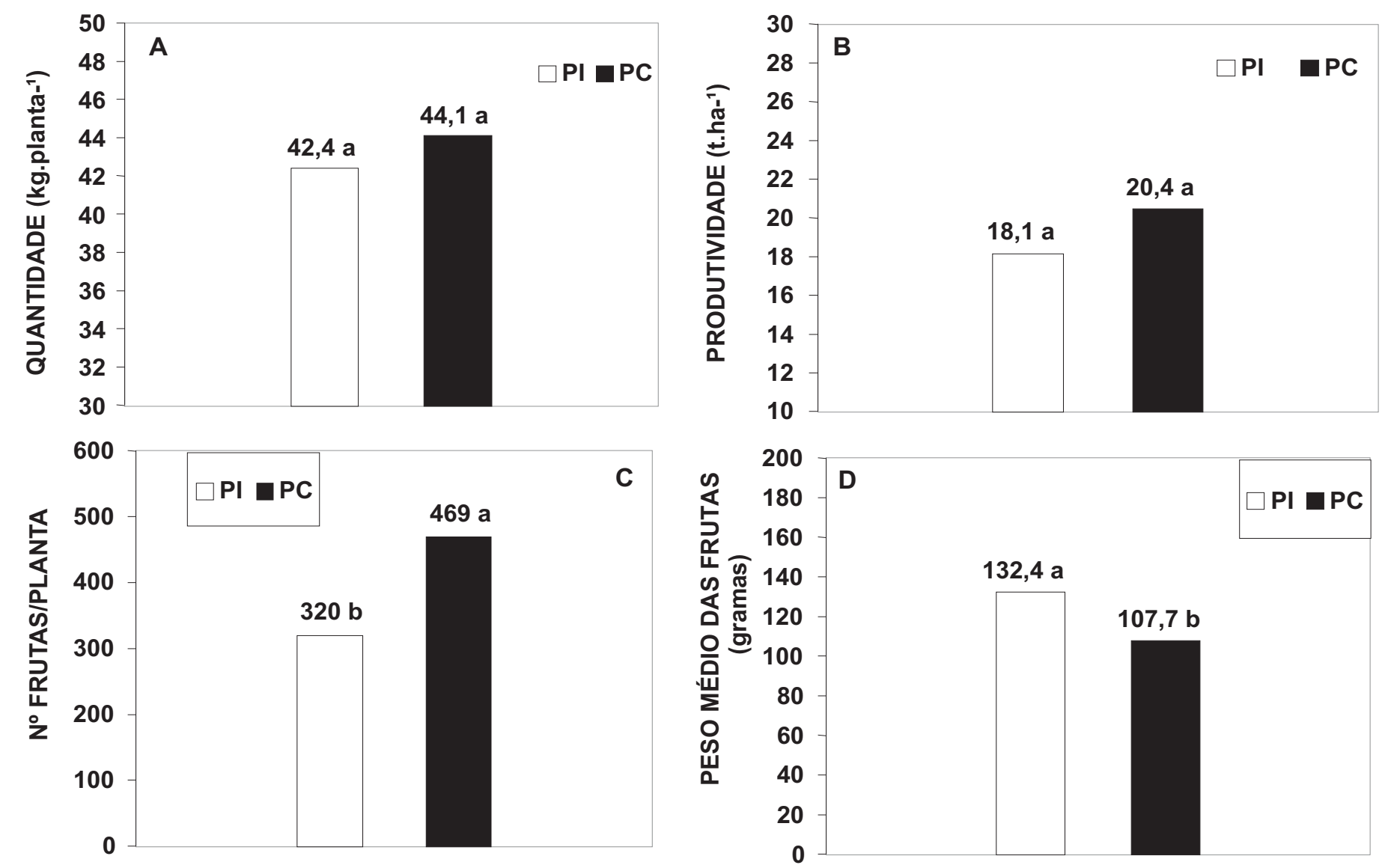

FIGURA 1 - Quantidade em kg.planta- ${ }^{1}$ (A), produtividade em t.ha- ${ }^{1}(B)$, número de frutas por planta (C) e peso médio das frutas em gramas (D), produzidas pelo pessegueiro cv. Marli, conduzido nos sistemas de PI e PC. São Jerônimo-RS, 2001. Médias seguidas da mesma letra não diferem, significativamente, pelo teste $\mathrm{t}(\mathrm{P} \leq 0,05)$.

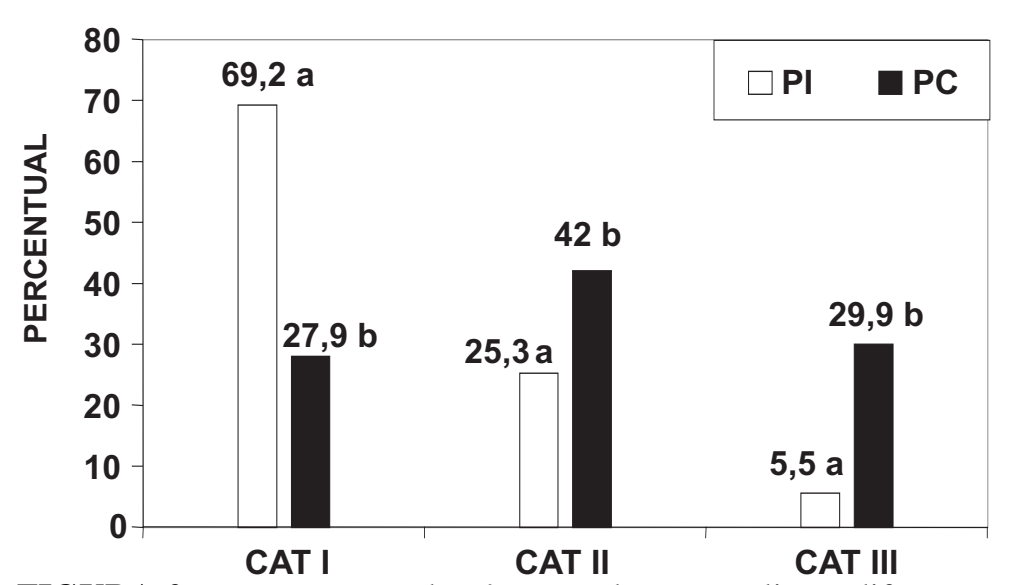

FIGURA 2 - Percentagem de pêssegos da cv. Marli em diferentes categorias: CAT I $(\geq 57 \mathrm{~mm})$, CAT II $(57 \mathrm{a} 48 \mathrm{~mm})$ e CAT III ( $\leq 48 \mathrm{~mm}$ ), produzidos em pomares conduzidos nos sistemas de PI e PC. São Jerônimo-RS, 2001. Médias seguidas da mesma letra não diferem, significativamente, pelo teste $\mathrm{t}(\mathrm{P} \leq 0,05)$. diferenças significativas entre os sistemas (Figura 3). Este fato pode ser explicado pelas condições meteorológicas do ano de 2001, que apresentou temperaturas mais elevadas, constantes e de inverno ameno (Tabela 1), as quais propiciaram condições semelhantes de crescimento e de maturação, mesmo com condições de manejo das plantas e do pomar mais favoráveis ao sistema PI. Estes dados diferem dos apresentados por Fachinello et al. (2002), que relatam que, na safra de 2001, na região de Pelotas, as frutas colhidas na PI apresentaram maior acidez do que as frutas colhidas na PC.

Não foram constatadas diferenças significativas nos teores de sólidos solúveis totais (SST) dos pêssegos produzidos em ambos os sistemas (Figura 3). Diferenças significativas somente seriam esperadas em condições meteorológicas extremantente diferentes entre as áreas. Como as áreas eram muito próximas entre si, as condições ambientais eram muito semelhantes, com temperaturas elevadas e com um período de estiagem próximo à colheita (Tabela 1). Estes dados diferem dos obtidos por Fachinello et al. (2002), que relatam que as frutas produzidas na safra de 2001, na região de Pelotas, apresentaram menores teores de SST no sistema de PI em relação à PC. 

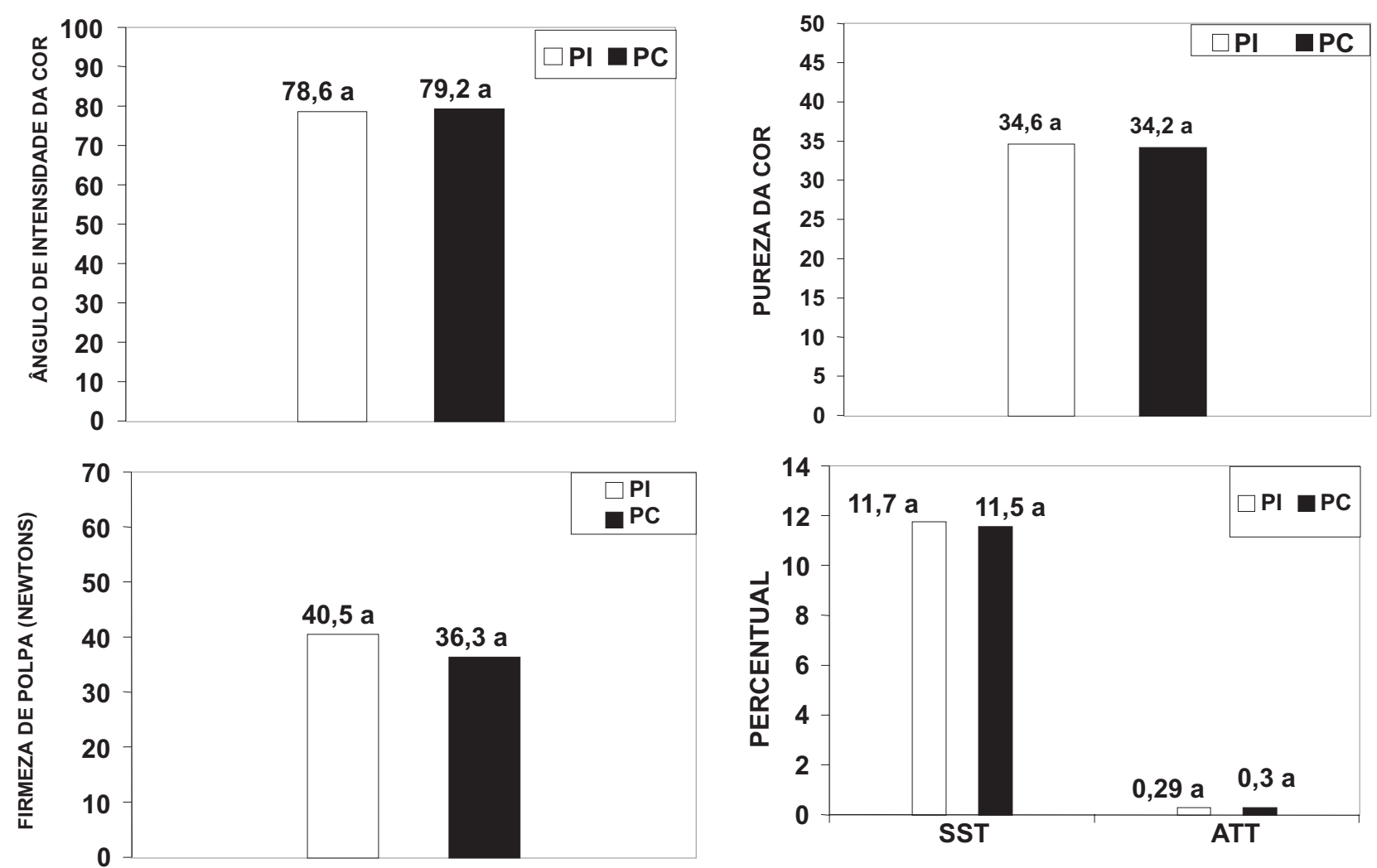

FIGURA 3 - Intensidade (A) e pureza (B) da cor vermelha da superfície dos pêssegos, firmeza de popa (C), \% de SST e \% ATT da cv. Marli (D), produzidos em pomares conduzidos sob sistemas de PI e PC. São Jerônimo-RS, 2001. Médias seguidas da mesma letra não diferem, significativamente, pelo teste $\mathrm{t}(\mathrm{P} \leq 0,05)$.

\section{CONCLUSÕES}

1) O pomar conduzido no sistema de PI apresentou produção semelhante ao sistema de PC, mas com frutas de maior peso e calibre.

2) A maioria das frutas produzidas no sistema de PI foi classificada como CAT I ( $\geq 57 \mathrm{~mm}$ ), enquanto no sistema de PC a maioria das frutas foi classificada como CAT II (de 48 a $57 \mathrm{~mm})$ e CAT III $(\leq 48$ $\mathrm{mm})$.

3) Os sistemas de produção não afetaram a acidez total titulável, os sólidos solúveis totais, a cor e a firmeza dos frutos da cv. Marli.

\section{REFERÊNCIAS}

BOTTON,M.; GARRIDO,L.R.; GIRARDI, C.L.; HOFFMANN,A.; MELO, G.W.B.; BERNARDI, J.; SÔNEGO, O.; CZERMAINSKI, R.D. Avaliação do sistema de produção integrada de pêssego de mesa na serra do RS - safra 1999/2000. In: SEMINÁRIO SOBRE PRODUÇÃO INTEGRADADEFRUTAS DECLIMATEMPERADO NO BRASIL, 2., 2000, Bento Gonçalves. Anais... Bento Gonçalves: Embrapa Uva e Vinho, 2000. p. 64-77.

FACHINELLO, J. C.; GRUTZMACHER, A. D.; HERTER, F. G.; CANTILLANO, F.; MATTOS, M. T.; FORTES, J.F.; AFONSO, A. P.; TIBOLA, C. S. Avaliação do sistema de produção integrada de pêssego de conserva na região de Pelotas-safra 1999-2000. In: SEMINÁRIO SOBRE PRODUÇÃO INTEGRADADE FRUTAS, 2. 2000, Bento Gonçalves. Anais... Bento Gonçalves: Embrapa Uva e Vinho, 2000. p. 78-85.

FACHINELLO, J.C.; BOTTON, M.; MARODIN, G. A. B.; COUTINHO, E. F. Produção integrada de pêssegos no Rio Grande do Sul: situação atual e perspectivas. In: SEMINÁRIO SOBRE PRODUÇÃO INTEGRADADE FRUTAS, 3., 2001, Bento Gonçalves. Anais... Bento Gonçalves: Embrapa Uva e Vinho, 2001. p. 42-47.

FACHINELLO, J. C.; TIBOLA, C. S.; VICENZI, M.; PARISOTTO, E.;
PICOLOTTO, L.; MATTOS, M. L. T. Produção Integrada de Pêssegos na Região de Pelotas - RS. In: SEMINÁRIO SOBRE PRODUÇÃ̃O INTEGRADADE FRUTAS, 4., 2002, Bento Gonçalves. Anais... Bento Gonçalves: Embrapa Uva e Vinho, 2002.p. 71.

FARIAS, R. M. Produção Convencional x Integrada em pessegueiro na Depressão Central do Rio Grande do Sul. 2002. 100f. Dissertação (Mestrado em Fitotecnia) - Faculdade de Agronomia, Universidade Federal do Rio Grande do Sul, Porto Alegre, 2002.

MARODIN, G.A.B.; SARTORI, I.A.; Situação das frutas de caroço no Brasil e no mundo. In: SIMPÓSIO INTERNACIONAL DE FRUTAS DECAROÇO: PÊSSEGOS, NECTARINAS EAMEIXAS, 2000, Porto Alegre. Anais... Porto Alegre: Universidade Federal do Rio Grande do Sul, 2000. p. 7-16.

NACHTIGALL, G.R.; GIRARDI, C.L.; BOTTON, M.; MARODIN, G.; HOFFMANN. A.; MELO, G.W.B.; SÔNEGO, O.R.; BERNADI, J.; FACHINELLO, J.C.; HERTER, F.G. Produção Integrada de Frutas de Caroço. In: SIMPÓSIO INTERNACIONAL DE FRUTAS DE CAROÇO: PÊSSEGOS, NECTARINAS E AMEIXAS, 2000, Porto Alegre. Anais... Porto Alegre: Universidade Federal do Rio Grande do Sul, 2000. p.61-71.

NORMAS de Produção Integrada de Pêssego (PIP): versão II. Pelotas: Ed. UFPEL/EMBRAPA/UFRGS/URCAMP, 2001.52p.

RASEIRA,A.; PEREIRA, J.F.M.; MEDEIROS, A.R.M.; CARVALHO, F.L.C. Instalação e manejo do pomar. In: MEDEIROS, C.A.B.; RASEIRA, M. do C.B. A cultura do pessegueiro. Brasília: Embrapa - SPI, 1998. p. $130-160$.

SARTORI, I. A. Aplicação de auxinas e incisão anelar em pessegueiro cv. Diamante. 2001. 111f. Dissertação (Mestrado em Fitotecnia) Faculdade de Agronomia, Universidade Federal do Rio Grande do Sul, Porto Alegre, 2001.

SEIBERT, E. Efeitos de pulverizações de ethepon na maturação e frigoconservação de peras cv. Packham's triumph. 1997. 100f. Dissertação (Mestrado em Fitotecnia) - Faculdade de Agronomia, Universidade Federal do Rio Grande do Sul, Porto Alegre, 1997. 